\title{
Magnified Expansion and Compression of Subpicosecond Pulses from a Frequency- Doubled Nd:YLF Laser
}

\author{
OSCAR E. MARTÍNEZ, PRABHURAM THIAGARAJAN, MARIO C. MARCONI, \\ AND JORGE J. ROCCA, MEMBER, IEEE
}

\begin{abstract}
Pulses from a mode-locked frequency-doubled Nd:YLF laser have been spectrally broadened and subsequently expanded to more than $200 \mathrm{ps}$ and recompressed to 750 fs by grating compressors with magnifying telescopes. The new design magnifies the time delay dispersion to a factor 10 times larger than achievable with standard compressors of similar size. The design of the system and its sensitivity to the variations in the position of its optical components is analyzed. The scheme will allow efficient amplification of subpicosecond pulses in dye amplifiers.
\end{abstract}

\section{INTRODUCTION}

$I^{\prime}$ $\mathrm{N}$ A recent paper [1] Martinez proposed the use of an expansion-amplification-recompression scheme by means of a grating pair with telescopes. The aim is to efficiently amplify femtosecond pulses, and is different from the chirped pulsed amplification of Strickland and Mourou [2] in that the primary purpose of the pulsewidth magnification is not to avoid the nonlinearities, but to enter in a quasi-steady-state amplification regime and also in that gratings are used in the expansion instead of fibers, allowing a better match with the grating compressor. This amplification regime permits one to overcome the limitations imposed by amplified spontaneous emission in a high-gain medium such as a dye amplifier, allowing for efficient energy transfer from the pump to the short pulse.

The magnification and sign change of the time delay dispersion by means of a telescope inside a compressor was already predicted and proposed for some applications by Martinez et al. [1], [3], [4], and it has only been used previously without magnification [5], [6]. Here we report the expansion of $35 \mathrm{ps}$ pulses to more than $200 \mathrm{ps}$ and its subsequent recompression using systems with magnified

Manuscript received March 9, 1989. This work was supported by N.S.F.-CONICET U.S.A./Argentina Cooperative Science Program under Grant INT 8802563, and by the National Science Foundation under Grants ECS 870507 and ECS 8606226 . M. C. Marconi was supported by a Fellowship from the Universidad Nacional de Buenos Aires and Consejo Nacional de Investigaciones Cientificas y Tecnicas de la Republica Argentina.

O. E. Martinez is with the Division Fisica del Solido, Comision Nacional de Energia Atomica, Libertador 8250-1429, Buenos Aires, Argentina.

P. Thiagarajan, M. C. Marconi, and J, J. Rocca are with the Department of Electrical Engineering, Colorado State University, Fort Collins, CO 80523.

IEEE Log Number 8930152 dispersion designed according to the previously reported equations [7]. Pulses from a mode-locked frequency-doubled neodymium YLF lasers were expanded and compressed yielding a pulsewidth within 15 percent of their transform limited value of $660 \mathrm{fs}$. This work is to our knowledge the first time that the full capability of the scheme is used not only to adjust the sign, but also to magnify the dispersion.

\section{Description of the Magnified Compressor}

A scheme of the magnified compressor is given in Fig. 1. The system consists of a grating pair with a three-lens telescope inserted between them. The design equations for the grating expansor and compressor are obtained using the matrix formalism presented by Martinez [7], which also allows the determination of the sensitivity of the performance of the system to errors in the positioning of its optical components.

A double transit through the grating pair is required in order to compensate for lateral displacement of the different frequencies [8], [9]. Following the procedure given in [7] the matrix must be computed after each dispersive element, retaining the corresponding phase term $\phi_{3}$. Assuming that the input beam has no initial angular nor lateral dispersion, the first term to be retained is after the first reflection on the second grating. The $3 \times 3$ matrix for that first transit is then

$$
\begin{aligned}
\left(\begin{array}{lll}
A & B & E \\
C & D & F \\
0 & 0 & 1
\end{array}\right)= & \left(\begin{array}{ccc}
\frac{1}{\alpha_{2}} & 0 & 0 \\
0 & \alpha_{2} & -\alpha_{2} F_{2} \\
0 & 0 & 1
\end{array}\right)\left(\begin{array}{ccc}
A^{\prime} & B^{\prime} & 0 \\
C^{\prime} & D^{\prime} & 0 \\
0 & 0 & 1
\end{array}\right) \\
& \left(\begin{array}{ccc}
\alpha_{1} & 0 & 0 \\
0 & \frac{1}{\alpha_{1}} & F_{1} \\
0 & 0 & 1
\end{array}\right)
\end{aligned}
$$

where

$$
F_{i}=\frac{\lambda^{2} \Delta w}{2 \pi c d_{i} \cos \theta_{i}} \quad \alpha_{i}=\frac{\cos \theta_{i}}{\cos \gamma_{i}}
$$




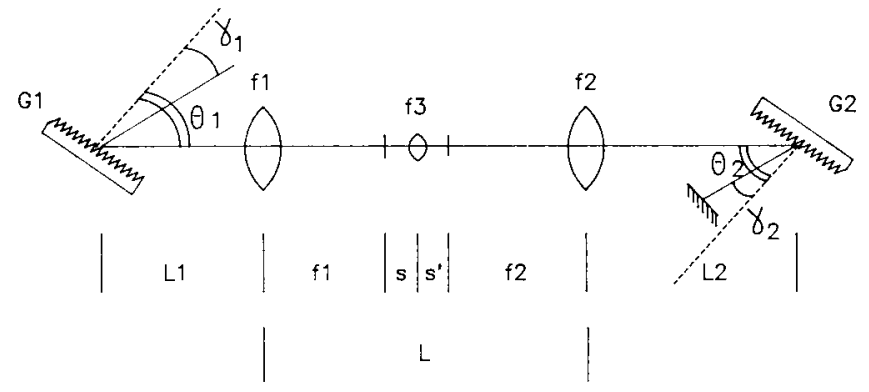

Fig. 1. Grating compressor with magnifying telescope.

$c$ is the speed of light in vacuum, $d_{i}$ is the groove spacing of the grating $G_{i}, \lambda$ is the central wavelength, and the angles $\gamma_{1}, \gamma_{2}, \theta_{1}$, and $\theta_{2}$ are shown in Fig. 1. $\mathbf{A}^{\prime}, \mathrm{B}^{\prime}, \mathrm{C}^{\prime}$, and $\mathrm{D}^{\prime}$ are the elements of the $2 \times 2$ matrix describing the propagation between the two gratings, and in this case are given by

$$
\begin{aligned}
A^{\prime}= & -\left(f_{2}-L_{2}\right) \frac{\delta}{f_{1} f_{2}}-\frac{f_{2}}{f_{1}}\left(1-\frac{s}{f_{3}}\right) \\
B^{\prime}= & -\frac{f_{1} f_{2}}{f_{3}}+\left(f_{1}-L_{1}\right) \frac{f_{2}}{f_{1}}\left(1-\frac{s}{f_{3}}\right)+\left(f_{2}-L_{2}\right) \frac{f_{1}}{f_{2}} \\
C^{\prime}= & \frac{\delta}{f_{1} f_{2}} \\
D^{\prime}= & -\frac{f_{1}^{\prime}}{f_{2}}\left(1-\frac{s^{\prime}}{f_{3}}\right)+\frac{\delta\left(f_{1}-L_{1}\right)\left(f_{2}-L_{2}\right)}{f_{1} f_{2}} \\
\delta= & s+s^{\prime}-\frac{s s^{\prime}}{f_{3}}
\end{aligned}
$$

where $s$ is the distance from the focal plane of lens 1 to lens 3 and $s^{\prime}$ is the distance from lens 3 to the focal plane of lens 2 . The propagation matrix for one transit is

$$
\left(\begin{array}{lll}
A & B & E \\
C & D & F \\
0 & 0 & 1
\end{array}\right)=\left(\begin{array}{lll}
\frac{\alpha_{1}}{\alpha_{2}} A^{\prime} & \frac{B^{\prime}}{\alpha_{1} \alpha_{2}} & \frac{B^{\prime} F_{1}}{\alpha_{2}} \\
\alpha_{1} \alpha_{2} C^{\prime} & \frac{\alpha_{2}}{\alpha_{1}} D^{\prime} & \alpha_{2}\left(D^{\prime} F_{1}-F_{2}\right) \\
0 & 0 & 1
\end{array}\right) .
$$

The phase term to be retained after the first pass through the compressor is [7]

$$
\phi_{3}=\frac{k}{2} F_{i} x_{i+1}=-\frac{k}{2} \alpha_{2} F_{2} E
$$

where $F_{i}$ is the $F$ element of the matrix for the optical element $i$ causing the dispersion, in this case grating $G_{1}$, and $x_{i+1}$ is the $x$ displacement after this element. For the beam to be reflected back over itself, it must carry no residual angular dispersion after this first transit, i.e., one must require $F=0$, which yields

$$
\left(D^{\prime} F_{1}-F_{2}\right)=0 \text {. }
$$

Once this condition is fulfilled, the second reflection in $G_{2}$ will introduce a phase term identical to the first one. This is so because the lateral displacement multiplied by the angular dispersion of the grating is conserved upon the inversion of the optical path (the product of the coefficients $A$ and $F$ of a grating does not change upon exchange of the input and output angles). Hence the phase term arising from this two reflections is just twice that given in (9):

$$
\phi_{3}=-k \alpha_{2} F_{2} E=-k F_{1} F_{2} B^{\prime} .
$$

The matrix $M_{2,1}$ for the return transit between the second grating and the first one can be obtained by exchanging subscript 1 with 2 and $s^{\prime}$ with $s$ in the elements of the matrix $A^{\prime} B^{\prime} C^{\prime} D^{\prime}$ given in (3)-(6), and results in

$$
M_{2,1}=\left(\begin{array}{lll}
D^{\prime} & B^{\prime} & 0 \\
C^{\prime} & A^{\prime} & 0 \\
0 & 0 & 1
\end{array}\right) .
$$

The matrix $M_{2,1}$ for the return transit between the second grating and the first one can be obtained by exchanging subscript 1 with 2 and $s^{\prime}$ with $s$ in the elements of the matrix $A^{\prime} B^{\prime} C^{\prime} D^{\prime}$ given in (3)-(6), and results in

$$
\begin{aligned}
& \left(\begin{array}{lll}
a & b & e \\
c & d & f \\
0 & 0 & 1
\end{array}\right) \\
& =\left(\begin{array}{ccc}
1-2 A^{\prime} D^{\prime} & \frac{-2 B^{\prime} D^{\prime}}{\alpha_{1}^{2}} & \frac{2}{\alpha_{1}} B^{\prime}\left(F_{2}-D^{\prime} F_{1}\right) \\
-2 \alpha_{1}^{2} A^{\prime} C^{\prime} & 1-2 A^{\prime} D^{\prime} & 2 \alpha_{1} A^{\prime}\left(F_{2}-D^{\prime} F_{1}\right) \\
0 & 0 & 1
\end{array}\right) .
\end{aligned}
$$

As no residual angular dispersion is desired, $f=0$ must be imposed, which is fulfilled when (10) is satisfied, yielding at the same time no lateral dispersion $(e=0)$. Equation (10) can be satisfied to first order in frequency shift by adjusting $D^{\prime}$ in order to compensate for any difference between $F_{1}$ and $F_{2}$, but it can only be satisfied to all orders in frequency shift if

$$
\begin{aligned}
& F_{1}=F_{2} \\
& D^{\prime}=1
\end{aligned}
$$

(for example, see higher order terms for $F_{1}$ in [7]).

Then, from (11), (14), and (15)

$$
\phi_{3}=-k B^{\prime} F_{1}^{2} \text {. }
$$


As $e=f=0$ and there is no input angular nor lateral dispersion, $x_{\mathrm{in}}=x_{\mathrm{in}}^{\prime}=0$, no output dispersion results, $x_{\text {out }}=x_{\text {out }}^{\prime}=0$, and hence the phase term given in (16) is the only significant one for the computation of the time delay dispersion. This is so as long as the beam is well collimated, as discussed by Martinez in [9]. The time delay dispersion will be given by

$$
\frac{d T}{d w}=\frac{d^{2} \phi_{3}}{d w^{2}}=-k B^{\prime} \frac{d^{2}\left(F_{1}^{2}\right)}{d w^{2}}
$$

which to first order gives a broadened pulsewidth $T_{b}$ given by

$$
T_{b} \cong\left|k B^{\prime} \frac{d^{2}\left(F_{1}^{2}\right)}{d w^{2}}\right| \frac{2 \pi}{T_{0}}=K T_{0}
$$

where

$$
T_{0}=\frac{2 \pi}{\Delta w}
$$

and $K$ is the compression factor for the compressor or expansor. Equation (16) gives a phase term similar to that obtained with a standard two-grating compressor, but with a factor $B^{\prime}$ instead of the grating separation. The coefficient $B^{\prime}$ then is the equivalent grating separation, and from (4), (6), and (15):

$$
B^{\prime}=L_{2}+L_{1} \frac{f_{2}}{f_{1}}\left(\frac{s}{f_{3}}-1\right)-\frac{f_{2}}{f_{3}}\left(f_{1}+s\right) .
$$

tained operating the laser at $1.3 \mathrm{~W}$ and corresponded to a pulsewidth of $490 \mathrm{fs}$.

The compressor used to expand the pulse had $f_{1}=f_{2}=$ $50, f_{3}=2.5, L_{1}=21$, and $L_{2}=26 \mathrm{~cm}$ and a 1800 lines $/ \mathrm{mm}$ holographic grating used at an angle close to Littrow incidence $\left(\alpha_{1}=1, F_{1}=0.3\right.$ fs $\left.\Delta w\right)$. From the conditions $\delta=0$ and $f_{1} / s=f_{2} / s^{\prime}$ we obtained $s=s^{\prime}=$ $2 f_{3}=5 \mathrm{~cm}$. The total length for the telescope is then $L$ $=110 \mathrm{~cm}$ and the magnification from (22) is $M=10$. From 21 the equivalent dispersion distance is then $B^{\prime}=$ $-10.53 \mathrm{~m}$. The pulse from the fiber already required a compression distance of about $3.5 \mathrm{~m}$, depending on the input power. Hence the final compressor was designed to compensate about $14 \mathrm{~m}$. This was achieved using $f_{1}=f_{2}$ $=1 \mathrm{~m}, f_{3}=-7 \mathrm{~cm}, L_{1}=26 \mathrm{~cm}$, and a similar pair of gratings. Then $s=s^{\prime}=-14 \mathrm{~cm}$ and $L=1.72 \mathrm{~m}$ resulted. The distance $L_{2}$ was adjusted in order to minimize the autocorrelation and resulted in $L_{2}=185 \mathrm{~cm}$ giving a total equivalent distance of $B^{\prime}=14.4 \mathrm{~m}$. Wings in the autocorrelation trace were canceled by spectral windowing at the position of the second grating in the expansor [10]. The pulse was reduced to an almost square spectrum of $10.6 \AA$ bandwidth, as shown in Fig. 2. The transformlimited pulse with that spectrum should have a FWHM of $660 \mathrm{fs}$, and we measured a $1 \mathrm{ps}$ autocorrelation trace, corresponding to $750 \mathrm{fs}$ assuming that the pulse shape corresponds to the Fourier transform of a square spectrum. The pulsewidth after the expansion could not be accu- rately measured due to the limited window of our autocorrelator ( $100 \mathrm{ps})$, but could be estimated to be larger than 200 ps from the slopes of the autocorrelation signal. The expected broadening of a $10.6 \AA$ bandwidth, square spectrum pulse is

$$
\Delta T=\frac{d t}{d w} \Delta w=\frac{d^{2} \phi_{3}}{d w^{2}} \Delta w=216 \mathrm{ps}
$$

which is consistent with our measurements.

The maximum broadening achievable depends on the grating size, not on the bandwidth [1], and is obtained by the condition that all the grating is covered by the pulse spectrum. The grating size limits the grating separation. For the particular case for which the telescope is focused to the infinite, that is $\delta=0$, and from (6), (7), (15), and (20):

$$
B^{\prime}=L_{1}+L_{2}-M L
$$

where $L$ is the separation between the lenses 1 and 2 , and

$$
M=\frac{f_{2} f_{1}}{f_{3}\left(f_{1}+f_{2}\right)} .
$$

The equivalent grating separation results from the contribution of the distance $L$ magnified by a factor $M$ plus the distances $L_{1}$ and $L_{2}$ that have no magnification because they correspond to a region of normal dispersion. Notice that the factor $M$ can have either sign depending on the sign of $f_{3}$, providing negative distances when needed.

The simplified case discussed above of focusing the telescope to infinite, could have been analyzed in a similar manner as done for the two lenses telescope by Martinez et al. [3]. The more complete analysis presented here shows that the condition of focusing to infinite is not necessary, thus reducing the numbers of parameters to be adjusted in the telescope. In fact, in order to accomplish the condition given in (10), only one parameter $s$ or $s^{\prime}$, must be adjusted while the simplified case would require the adjustment of both $s$ and $s^{\prime}$. Moreover, to first order in frequency shift the adjustment could be done by tilting the grating and so changing $F_{2}$. No fine tuning of either $s$ or $s^{\prime}$ would be needed in this case.

Another advantage of the formalism used is that it allows the determination of the distortions arising from positioning errors with any of the optical components. As will be shown, this is particularly important with systems with large compression factors.

We will restrict our discussion to the most important distortion source, which is the presence of a residual angular dispersion after propagating through the system. Such angular dispersion would give rise to further time delay dispersion $T_{\text {res }}$ as the pulse propagates away from the compressor, given by [9]

$$
\frac{d T_{\mathrm{res}}}{d w}=k z\left(\frac{d \theta}{d w}\right)^{2}=k z\left(\frac{d f}{d w}\right)^{2}
$$




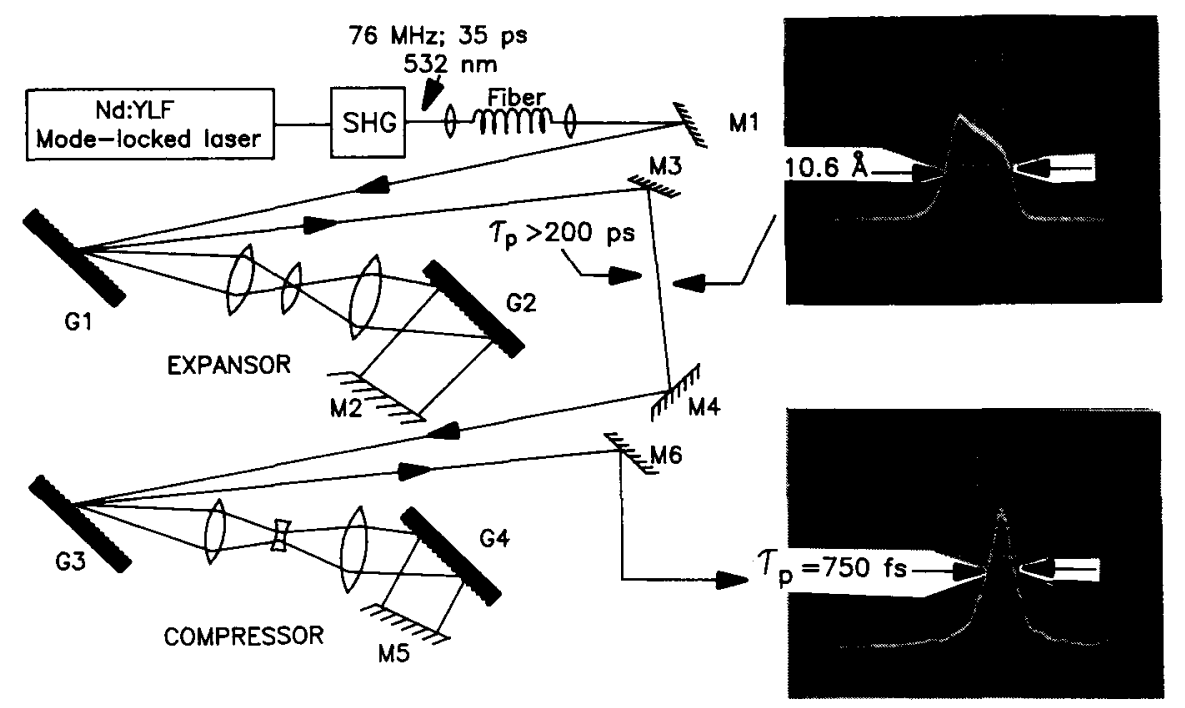

Fig. 2. Schematic representation of the experimental setup used to expand and recompress the pulses from a mode-locked frequency-doubled Nd: YLF laser. $M_{1}-M_{6}$ are high reflector mirrors and $G_{1}-G_{4}$ are 1800 lines $/ \mathrm{mm}$ gratings. The top oscilloscope trace shows the spectrum of the pulse. The horizontal scale is $4.3 \AA$ /division. The bottom oscilloscope photograph displays the autocorrelation trace of the pulse at the output of the compressor.

where $T_{\text {res }}$ is the residual time delay, $z$ is the propagation distance and $f$ is the matrix element given in (13).

One way that residual dispersion can appear is that (14) is not satisfied. This condition is similar to that required for a standard two-grating compressor: that the gratings must be aligned parallel to each other. The other possibility is that (15) is not satisfied. $D^{\prime}$ can be adjusted by moving lens 2 to change $s^{\prime}$ or else moving lens 3 changing at the same time $s$ and $s^{\prime}$, but keeping constant $s+s^{\prime}$. In both cases the dominant term for the variation of $D^{\prime}$ results:

$$
\Delta D^{\prime}=\Delta s^{\prime} \frac{d D^{\prime}}{d s^{\prime}} \cong \frac{f_{1}}{f_{2} f_{3}} \Delta s^{\prime}
$$

and the residual time delay dispersion is obtained from (23) using (13) and (24).

$$
\frac{d T_{\mathrm{res}}}{d w}=\frac{k B^{\prime}}{m}\left(\frac{d^{2} F_{1}^{2}}{d w^{2}}\right)
$$

where

$$
m=\left(\frac{f_{3} f_{2}}{2 \alpha_{1} f_{1} \Delta s^{\prime}}\right)^{2} \frac{B^{\prime}}{z} .
$$

If this additional broadening is to be made negligible, one must require that the broadening induced is smaller than $T_{0}$, that is

$$
\Delta T_{\mathrm{res}}=\frac{d t}{d w} \Delta w<T_{0}
$$

and using (18) and (25)

$$
m>K
$$

The mismatch $\Delta D^{\prime}=D^{\prime}-1$ will also give rise to lateral spatial dispersion. With a similar analysis the lat- eral displacement observed after the double pass through the compressor $\left(\Delta x_{1}\right)$ can be compared with that after the second grating $\left(\Delta x_{2}\right)$, yielding

$$
\frac{\Delta x_{1}}{\Delta x_{2}}=2 \Delta D^{\prime}=2 \alpha_{2} \frac{f_{1}}{f_{2} f_{3}} \Delta s^{\prime}
$$

and from (26), (28), (29), and as $\alpha_{1}=\alpha_{2}$

$$
\left(\frac{\Delta x_{2}}{\Delta x_{1}}\right)^{2}>\frac{K z}{B^{\prime}} \text {. }
$$

The parameter $D^{\prime}$ can then be adjusted while observing that the lateral distortion is reduced below the value given by (30).

\section{EXPERIMENTAL RESUlts}

Using the formalism given above, two compressors were designed. The first one is to be used as an expansor to broaden a short pulse to be amplified and the second one is for the compression after amplification. The experimental setup is shown in Fig. 2. The 35 ps pulses from a frequency-doubled Nd:YLF laser were spectrally broadened by self-phase modulation in a $98 \mathrm{~m}$ long monomode polarization-preserving fiber. The spectrum could be broadened to up to $20 \AA$, depending on the output power. In order to reduce the damage risk of the KTP second-harmonic crystal, the power input in the fiber was reduced to $1 \mathrm{~W}$, yielding a broadening somewhat larger than $11 \AA$. The Raman component at the output had 30 percent of the total power and the fiber throughput was 20 percent. The pulsewidth at the fiber output was about 60 ps. The system in this first stage is similar to the one described by Johnson et al. [8], although we could not 
achieve shorter pulses due to the high losses of our fiber. The shortest pulses achieved with our system were obLet $D$ be the grating diameter, then it can be easily shown that for our gratings:

$$
\Delta T_{\max }=2 k\left(\frac{d \theta}{d w}\right)^{2} \Delta w B^{\prime}=\frac{2 \lambda D}{c d}=63.6 D \mathrm{ps} / \mathrm{cm} .
$$

It must be mentioned that our system was originally designed for a $20 \AA$ bandwidth that would have covered most of the $7.5 \mathrm{~cm}$ diameter of the second grating $G_{2}$ of the expansor.

Regarding the precision with which the lenses had to be positioned, from (24) and considering that the coefficient $m$ has to be greater than the compression factor (in this case about 300 ), and assuming a propagation $z=3 \mathrm{~m}$ away from the compressor it results $\Delta s^{\prime}<4 \mathrm{~mm}$. A similar consideration for the expansor yields $\Delta s^{\prime}<1 \mathrm{~mm}$. In fact, the alignment of the compressor was fairly simple while much more care had to be taken in the alignment of the expansor. This can be easily understood by noticing that even though the expansor had a shorter equivalent distance, it had a larger magnification. This adjustment was done observing the lateral distortion of the output beam $\Delta x_{1}$, which from (30) had to be kept below $6 \mathrm{~mm}$ for the compressor and $4 \mathrm{~mm}$ for the expansor.

\section{REFERENCES}

[1] O. E. Martinez. "Design of high-power ultrashort pulse amplifiers by expansion and recompression," IEEE J. Quantum Electron., vol. QE23, pp. 1385-1387, 1987.

[2] D. Strickland and G. Mourou. "Compression of amplified chirped optical pulses," Opt. Commun., vol. 56, pp. 219-221, 1985.

[3] O. E. Martinez, J. P. Gordon, and R. L. Fork. "Negative group velocity dispersion using refraction," J. Opt. Soc. Amer. A, vol. 1, pp. 1003-1006, 1984

[4] O. E. Martinez. "3000 times grating compressor with positive group velocity dispersion: Application to fiber compensation in $1.3-1.6 \mu \mathrm{m}$ Region," IEEE J. Quantum Electron., vol. QE-23, pp. 59-64, 1987.

[5] M. Pessot, P. Maine, and G. Mourou. "1000 times expansion/ compression of optical pulses for chirped pulse amplification," Opt. Commun., vol. 62, pp. 419-421, June 1987.

[6] A. M. Weiner, J. P. Heritage, and J. A. Salehi. "Encoding and decoding of femtosecond pulses," Opt. Lett., vol. 13, pp. 300-302, 1988.
[7] O. E. Martinez, "Matrix formalism for pulse compressors, " IEEE J. Quantum Electron., vol. 24, p. 2530, 1988.

[8] A. M. Johnson, R. H. Stolen, and W. M. Simpson, " $80 \times$ single stage compression of frequency doubled $\mathrm{Nd}$ : yttrium aluminum garnet laser pulses," Appl. Phys. Lett., vol. 44, pp. 729-731, 1984.

[9] O. E. Martinez. "Grating and prism compression in the case of finite beam size,' J. Opt. Soc. Amer., B, vol. 3, pp. 929-932, 1986.

[10] J. P. Heritage, R. N. Thurston, W. J. Tomlinson, A. M. Weiner, and R. H. Stolen, "Spectral windowing of frequency modulated optical pulses in a grating compressor," Appl. Phys. Lett., vol. 47, pp. 8789,1985 .

Oscar E. Martínez, for a photograph and biography, see p. 300 of the March 1989 issue of this Journal.

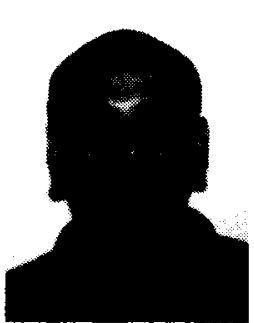

Prabhuram Thiagarajan was born in Coimbatore, India, on October 9,1965 . He received the Diploma in mechanical engineering from PSG Polytecnic in 1983 and the B.S. degree in electrical engineering from Colorado State University, Fort Collins, in 1988.

He is currently working towards the M.S. degree at Colorado State University. His main research is in the field of short pulse lasers and amplification techniques.

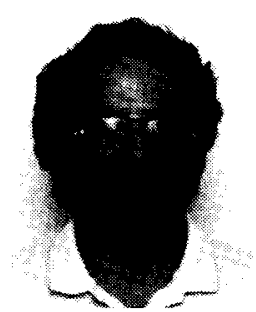

Mario C. Marconi was born in Buenos Aires, $\mathrm{Ar}-$ gentina, on March 7, 1955. He received the degrees of Licenciado en Fisica and Doctor en Fisica from the Universidad de Buenos Aires in 1980 and 1985 , respectively.

He joined CEILAP (CITEFA) in 1978, where he worked in new techniques to obtain short pulse emission in solid-state lasers. Since 1986 he has been with Colorado State University, Fort Collins, under a Postdoctoral Fellowship from the Universidad de Buenos Aires and the Consejo Nacional de Investigaciones Cientificas y Tecnicas (CONICET).

Jorge J. Rocca (S'80-M'83), photograph and biography not available at the time of publication. 